\title{
New derivation method and simulation of skin effect in biological tissue
}

\author{
Xiaoli Fan ${ }^{\mathrm{a}}$, Qianxiang Zhou ${ }^{\mathrm{a}, *}$, Zhongqi Liu ${ }^{\mathrm{a}}$ and Fang Xie ${ }^{\mathrm{b}}$ \\ ${ }^{a}$ School of Biological Science and Medical Engineering, Beihang University, No. 37, Xueyuan Road, \\ Haidian District, Beijing 100191, China \\ ${ }^{b}$ China North Vehicle Research Institute, Beijing 100072, China
}

\begin{abstract}
Based on the electrical properties of biological tissues, bioimpedance measurement technology can be employed to collect physiologic and pathologic information by measuring changes in human bioimpedance. When an alternating current (AC) is applied as a detection signal to a tissue, the current field distribution, which is affected by skin effect, is related to both the bioimpedance of the tissue and the AC frequency. These relations would possibly reduce the accuracy and reliability of the measurement. In this study, an electromagnetic theory-based method, in which cylindrical conductor were divided into layers, was used to obtain current field distribution models of human limbs. Model simulations were conducted in MATLAB. The skin effect phenomenon and its characteristics in human tissues at different frequencies were observed, thus providing essential data on skin effect, which are useful in the development of bioimpedance measurement technology.
\end{abstract}

Keywords: Skin effect, bioimpedance measurement technology, current density, conductivity

\section{Introduction}

Bioimpedance measurement is a non-invasive testing technology that can obtain biomedical information of human physiology and pathology by measuring the electrical characteristics of human tissues or organs $[1,2]$. When stimulating electrodes are placed on the surface of tissue and a small alternating current (AC) signal is injected, impedance of the tissues can be calculated by current and voltage signals received by the detection electrodes. The impedance data obtained using the noninvasive, safe, and easy-to-operate bioimpedance measurement technology can be used in various medical applications, such as cell measurement, tissue construction and composition analysis, and electrical impedance tomography (EIT) [3].

Skin effect was first proposed by Horace Lamb in 1883 and was exclusively applied to spherical metallic conductors. Oliver Heaviside further developed the theory that skin effect exists in conductors of all shapes [4]. It was well known that electromagnetic fields decay upon entering conducting regions [5].When an applied AC flows through a conductor, an electromagnetic field is generated because of changes in current direction. The repulsive force from the electromagnetic field induces

\footnotetext{
* Address for correspondence: Qianxiang Zhou, School of Biological Science and Medical Engineering, Beihang University, No. 37, Xueyuan Road, Haidian District, Beijing 100191, China. Tel.: 010-82338696; Fax: 010-82338696; Email: zqxg@buaa.edu.cn.
} 
electric charges to move toward the conductor's surface, leading to an uneven distribution of the current density, resulting in a change in the bioimpedance of the conductor [6,7]. Higher $\mathrm{AC}$ frequency leads to a more obvious effect.

Impedance rheogram technology and EIT are the two most important applications of bioimpedance measurement technology [8]. The principle of impedance rheogram technology is as follows: when a high-frequency AC is applied in certain areas of the human body, the bioimpedance value of the measured part changes, which is affected by vessel volume changes caused by heartbeat [9]. Corresponding changes in the blood flow can be obtained by measuring impedance changes. However, if skin effect is generated by AC in the biological tissue, then only impedance changes of the tissue's surface can be measured, which could not reflect internal physiological state. EIT produces images of biological tissues or organs based on the variation in impedances of biological tissues [10]. In EIT, AC is applied to biological tissues by stimulating electrodes and the voltage of the cross-section of the tissue is determined by detection electrodes. By considering the voltage around the cross-section as boundary conditions, the spatial distribution of conductivity can be calculated by finite element analysis iteration, forming a mathematical inverse question. Given the existence of skin effect, the distribution of the current field is not only related to impedance distribution of the tissue, but also affected by the induced current when AC reaches a threshold frequency. Therefore, impedance imaging based on current field distribution will lose its desired function, and electrical conductivity of the tissue calculated by the boundary voltage will include errors contrasting to the actual value; such imaging technique is not reliable for clinical diagnosis [11].

Although skin effect in a metal conductor has been established, available studies on the skin effect of biological tissues are insufficient. In general, metal conductors can be treated as homogeneous for skin effect research. However, the human body has a complex structure and cannot be regarded as a uniform conductor. Based on the electrical characteristics of human tissues, a method that divides cylindrical conductor into layers is used to calculate the current density distribution in human tissues. In this study, the skin effect phenomenon in human limbs is demonstrated at different AC frequencies.

\section{Theory and method}

\subsection{Electrical characteristics of human tissue}

Given the water content and relative density of human tissue, its conductivity is considered variable in different studies. Human body fluid, which contains a large number of charged particles, possesses strong electrical conductivity. Therefore, blood, lung, and muscle are all good conductors, whereas tissues with low fluid content, such as fat, bone, and skin, possess low electrical conductivity and dielectricity [12]. Tissues with strong electrical conductivity and dielectricity that are composed adjacently can be regarded as capacitor circuits [13]. Given the capacitance characteristics, the impendence values of human tissues depend on the frequency of stimulating current.

Studies showed that biological tissues remain safe and represent resistance characteristics under the action of a stimulating current with frequency ranging from $10 \mathrm{kHz}$ to $10 \mathrm{MHz}$. In general, bioimpedance measurement technology uses a stimulating AC with a frequency of $100 \mathrm{kHz}$ and strengths that are lower than $5 \mathrm{~mA}$, which satisfy the requirements of the measurement without harmful effect on the human body. Peter, et al. [14] provided accurate conductivity data through tissue resistivity measurement in a test tube, and Table 1 shows the conductivity data of different tissues at different frequencies under normal conditions. 
Table 1

Conductivity values of human tissues at different frequencies (all units in $\mathrm{S} / \mathrm{m}$ )

\begin{tabular}{lllllll}
\hline Tissue & $0 \mathrm{kHz}$ & $10 \mathrm{kHz}$ & $100 \mathrm{kHz}$ & $1 \mathrm{MHz}$ & $10 \mathrm{MHz}$ & $1 \mathrm{GHz}$ \\
\hline Muscle & 0.3 & 0.35 & 0.4 & 0.5 & 0.6 & 1.1 \\
Skin & 0.0007 & 0.004 & 0.06 & 0.3 & 0.4 & 1.2 \\
Fat & 0.022 & 0.023 & 0.023 & 0.024 & 0.025 & 0.0375 \\
Bone & 0.06 & 0.07 & 0.08 & 0.1 & 0.12 & 0.36 \\
\hline
\end{tabular}

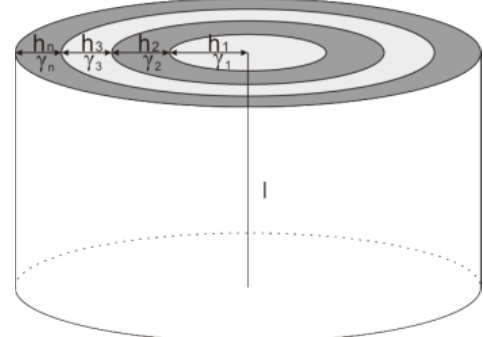

Fig. 1. Simplified model of human tissues.

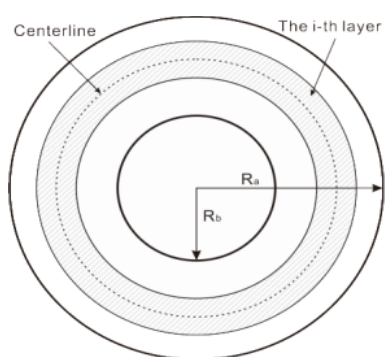

Fig. 2. Layering pattern of the model's cross-section.

\subsection{Simplified model of human tissues}

The combined form and conductivity of human tissue is simplified as a cylinder composed of several homocentric circles crossing the section. As shown in Figure 1, each circle represents one kind of tissue. $h_{1}, h_{2}, h_{3}$, and $h_{\mathrm{n}}$ represent the thickness of each tissue, with corresponding conductivities of $\gamma_{1}, \gamma_{2}, \gamma_{3}$, and $\gamma_{\mathrm{n}} ; l$ is the axial length.

\subsection{Simulation algorithm}

Analytical equations for cylindrical conductor are currently unavailable, and a numerical calculation method was used in this study. Finite element method was the most commonly solution, but the process is complicated and difficultly deals with a cylindrical conductor composed of concentric rings with different resistivities $[15,16]$. On the basis of elementary electromagnetic laws, a different solution that divides the cylindrical conductor into layers and treats each layer as a separate object is proposed in the current paper, thereby computing current density of each layer and obtaining the skin effect depth. The programming for this method is considerably simpler than that of finite element method; more divided layers produce more accurate results [17].

\subsubsection{Layering for conductor}

The simplified cylinder model is composed of several concentric rings. Therefore, rings representing different tissues can be layered further. Consider a ring conductor with an external radius of $R_{\mathrm{a}}$ and inner radius of $R_{\mathrm{b}} ; R_{\mathrm{b}}=0$ for solid conductor. An AC with frequency of $f$ is applied along the axial direction. Assume that the conductor is sufficiently long that the current density is axisymmetric and only related to the distance from the axis of the conductor. Hence, only the current distribution of the cross-section is studied here [18]. The cross-section of the cylindrical conductor is shown in Figure 2.

The cross-section is divided into $N$ concentric rings with the same thickness, $h$, which is expressed as $h=\left(R_{\mathrm{a}}-R_{\mathrm{b}}\right) / N$. The radius $r_{i}$ of the layer $i$ is the distance from the center of the cylindrical 
conductor to the center line of the layer $i$, which is expressed as $r_{i}=R_{\mathrm{b}}+(i-0.5) h$. When the cylinder is layered sufficiently, resulting in a sufficiently small thickness, the length of the center line of the layer $i$ can be set to the perimeter $c_{i}$, which is expressed as $c_{i}=2 \pi\left[R_{\mathrm{b}}+(i-0.5) h\right]$. In the calculation, given that the thickness of each layer is sufficiently small, the self-skin effect, which will not cause considerable errors, is disregarded.

\subsubsection{Formula derivation of numerical calculation}

When an AC is applied in a cylindrical conductor, the changes in magnetic flux inside and outside the conductor that are caused by changes in magnitude and direction of $\mathrm{AC}$ will generate induced electromotive force of different values in the layers. All ring conductors are in parallel and thus the induced voltage values in layers caused by external magnetic flux are equal. The following equations are the derivation of the calculation formula of internal mutual inductance and internal self-inductance between the layers caused by changes in internal magnetic flux.

We assume that the AC vectors applied from inside to outside of the conductor are respectively $\dot{I}_{1}, \dot{I}_{2}, \ldots, \dot{I}_{N}$, with corresponding root-mean-square (valid value) values of $I_{1}, I_{2}, \ldots, I_{N}$.

Magnetic fluxes generated by $I_{i}$ in layer $i, i+1 \ldots, N$ are all linked to the AC of layer $i$. In detail, a part of magnetic flux $\psi_{i i i}$ generated by $I_{i}$ in layer $i$ is linked to a part of $I_{i}$, which is expressed as $\psi_{i i i}=I_{i} \mu l \frac{h}{3 c_{i}}$. The magnetic fluxes in layer $i+1, i+2 \ldots, N$ generated by $I_{i}$ are all linked to $I_{i}$, which is expressed as $\psi_{k i i}=I_{i} \mu l \frac{h}{c_{k}}(k=i+1, i+2, \ldots, N)$. Therefore, the total flux linkage $\psi_{i i}$ can be expressed as Eq. (1), where $h$ is the thickness of the ring, $\mu$ is the magnetic permeability of the conductor, and $c_{i}$ is the perimeter of the layer $i$.

$$
\psi_{i i}=\sum_{k=i}^{N} \psi_{k i i}=I_{i} \mu l\left(\frac{h}{3 c_{i}}+\sum_{k=i+1}^{N} \frac{h}{c_{k}}\right)
$$

Moreover, internal self-inductance in layer $i$ can be obtained using Eq. (2).

$$
L_{i i}=\frac{\psi_{i i}}{I_{i}}=\mu l\left(\frac{h}{3 c_{i}}+\sum_{k=i+1}^{N} \frac{h}{c_{k}}\right)
$$

To calculate the mutual flux linkage and internal mutual inductance of layers $i$ and $j$, two situations should be considered: $i>j$ and $i<j$ were respectively calculated.

When $i>j$, leakage fluxes generated by $I_{j}$ in layer $i+1 \ldots, N$ are linked to $I_{i}$, which is expressed as $\psi_{k i j}=I_{j} \mu l \frac{h}{c_{k}}(k=i+1, i+2, \ldots, N)$. Meanwhile, the magnetic flux $\psi_{i i j}$ generated by $I_{j}$ in layer $i$ are linked to part of $I_{i}$, which is expressed as $\psi_{i i j}=I_{j} \mu l \frac{h}{2 c_{i}}$. Therefore, the total internal flux linkage $\psi_{i j}$ generated by $I_{j}$ and linked to $I_{i}$ can be expressed as:

$$
\psi_{i j}=I_{j} \mu l\left(\frac{h}{2 c_{i}}+\sum_{k=i+1}^{N} \frac{h}{c_{k}}\right)
$$

The internal mutual inductance is:

$$
M_{i j}=\frac{\psi_{i j}}{I_{j}}=\mu l\left(\frac{h}{2 c_{i}}+\sum_{k=i+1}^{N} \frac{h}{c_{k}}\right)
$$


When $i<j$, the magnetic fluxes generated by $I_{j}$ in layer $j+1 \ldots \ldots, N$ are linked to $I_{i}$, expressed as $\psi_{k i j}=I_{j} \mu l \frac{h}{c_{k}}(k=j+1, j+2, \ldots, N)$, whereas part of the magnetic flux $\psi_{j i j}$ generated by $I_{j}$ in layer $\mathrm{j}$ are linked to $I_{i}$, which is expressed as $\psi_{j i j}=I_{j} \mu l \frac{h}{2 c_{j}}$. Therefore, internal total flux linkage that is generated by $I_{j}$ and linked to $I_{i}$ can be expressed as:

$$
\psi_{i j}=I_{j} \mu l\left(\frac{h}{2 c_{j}}+\sum_{k=j+1}^{N} \frac{h}{c_{k}}\right)
$$

From Eq. (5), the internal mutual inductance can be obtained using:

$$
M_{i j}=\frac{\psi_{i j}}{I_{j}}=\mu l\left(\frac{h}{2 c_{j}}+\sum_{k=j+1}^{N} \frac{h}{c_{k}}\right)
$$

The voltage drop of layer $i$ includes self-resistance voltage drop, internal self-inductance and mutual inductance voltage drop, and induced voltage of the external magnetic flux. The sum of these voltage drops are equal to terminal voltage $U_{i}$, which is expressed as in the following equation:

$$
U_{i}=\sum_{k=1}^{i-1} j w M_{i k} I_{k}+\left(R_{i}+j w L_{i i}\right) I_{i}+j w M_{i(i+1)} I_{i+1}+\sum_{k=i+2}^{N} j w M_{i k} I_{k}+U_{0}
$$

$U_{0}$ represents the induced voltage caused by external magnetic flux, $\omega$ is the angular frequency of the AC current, which conforms to the equation $w=2 \pi f$, and $R_{i}$ gained by calculation is the direct current (DC) resistance value of layer $i$. Assuming that the conductivity of layer $i$ is $\gamma, R_{i}$ can be expressed as:

$$
R_{i}=\frac{1}{\gamma c_{i} h}
$$

Similarly, layer $i-1$ also conforms to Eq. (7):

$$
U_{i-1}=\sum_{k=1}^{i-2} j w M_{(i-1) k} I_{k}+\left(R_{i-1}+j w L_{(i-1)(i-1)}\right) I_{i-1}+j w M_{(i-1) i} I_{i}+\sum_{k=i+2}^{N} j w M_{(i-1) k} I_{k}+U_{0}
$$

From Eq. (6), we know that when $j>i, M_{i j}$ is only related to $j$ rather than $i$. Therefore,

$$
\sum_{k=i+1}^{N} M_{(i-1) k} I_{k}=\sum_{k=i+1}^{N} M_{i k} I_{k}
$$

All layers are parallel with each other, thus $U_{i}=U_{i-1}$. By combining Eqs. (7), (9), and (10), the following recurrence equation can be obtained:

$$
I_{i}=\frac{\sum_{k=1}^{i-2} j w\left(M_{(i-1) k}-M_{i k}\right) I_{k}+\left(R_{(i-1)}+j w L_{(i-1)(i-1)}-j w M_{i(i-1)}\right) I_{i-1}}{R_{i}+j w L_{i i}-j w M_{(i-1) i}}
$$

This recurrence equation is only applicable when $I \geq 3$. When $I=2$, a separate calculation is required: 


$$
I_{2}=\frac{\left(R_{1}+j w L_{11}-j w M_{21}\right) I_{1}}{R_{2}+j w L_{22}-j w M_{12}}
$$

On the basis of the above recursive equations, when $I_{1}$ is assumed, $I_{2}$ can be obtained by Eq. (12), and $I_{3}$ can be obtained by Eq. (11). Therefore, the AC value of each layer can be obtained using these recurrence equations. Similarly, the recursive Eq. (11) can be changed into the equation of $\dot{I}_{i}$ and $\dot{I}_{1}$. We learn that the modulus proportion relation between $\dot{I}_{i}$ and $\dot{I}_{1}$ is unrelated to the modulus value of $\dot{I}_{i}$. Therefore, $\dot{I}_{1}$ can be set to any number except 0 , without affecting AC distribution.

Then, we can calculate the valid values $I_{1}, I_{2}, \ldots, I_{N}$ of AC vectors $\dot{I}_{1}, \dot{I}_{2}, \ldots, \dot{I}_{N}$. The current density $J_{i}$ of layer $i$ can be obtained by density equation $J_{i}=I_{i} / S_{i}$, where $S_{i}$ is the cross-sectional area of the cylindrical layer $i$.

The above derivation shows that, at any given current value, corresponding equations are available for calculating other current values, that is, the current densities of layers are proportional. Therefore, the current values do not influence the skin effect, and the current value of the first layer can be optionally set.

\subsubsection{Algorithm validation}

The algorithm above is first verified by a metal conductor that is composed of a single material. Copper, with electrical conductivity of $5.8 \times 10^{7}$ and magnetic permeability of $4 \pi \times 10^{-7} \mathrm{H} / \mathrm{m}$, is the selected material. The outer diameter $\left(R_{\mathrm{a}}\right)$ is $20 \mathrm{~mm}$, and the inner diameter $\left(R_{\mathrm{b}}\right)$ is $15 \mathrm{~mm}$. The conductor is divided into 500 layers with $0.01 \mathrm{~mm}$ thickness for each layer. The current density of the outer layer and frequency are assumed to be $11 \mathrm{~A} / \mathrm{S}^{2}$ and $5 \mathrm{kHz}$, respectively.

When the electromagnetic wave propagates from the surface to the inside of the conductor, skin depth $d$ is defined as the passing distance when the electric field intensity or current density is $1 / e$ of the electric field intensity or current density of the surface [19]. The obtained current densities of the $408^{\text {th }}, 409^{\text {th }}$, and $410^{\text {th }}$ layers by input data and Eqs. (11), (12), and density equation, which are carried out in MATLAB, are $0.3649,0.3690$, and $0.3731 \mathrm{~A} / \mathrm{m}^{2}$, respectively. The $409^{\text {th }}$ layer possessed a value that most closely approximates the $1 / e$ of the surface, from which the skin depth can be calculated with the number of layers and layer thickness, that is, $d=(0.01 \mathrm{~mm}) \times(91)=0.91 \mathrm{~mm}$. Meanwhile, the value of skin depth obtained by Eq. (13) is $0.935 \mathrm{~mm}$. The two results obtained by different methods are basically the same, indicating the feasibility of the layer-dividing algorithm.

$$
\Delta=\sqrt{\frac{2}{\mu \gamma \omega}}
$$

Where $\Delta$ is the skin depth (m), $\mu$ is the magnetic permeability $(\mathrm{H} / \mathrm{m}), \gamma$ is the conductivity $(\mathrm{S} / \mathrm{m})$, and $\omega$ is the angular frequency $(\mathrm{rad} / \mathrm{s})$.

\section{Results and discussion}

Human limb was selected in this study, which is shaped like a cylinder, as a research object (Figure 2). From inside to outside, human limb consists of bone tissues, muscular tissues, fat, and skin [20], with corresponding conductivity of $\gamma_{1}, \gamma_{2}, \gamma_{3}$, and $\gamma_{4}$, and thickness of $h_{1}, h_{2}, h_{3}$, and $h_{4}$, as shown in Figure 3. 


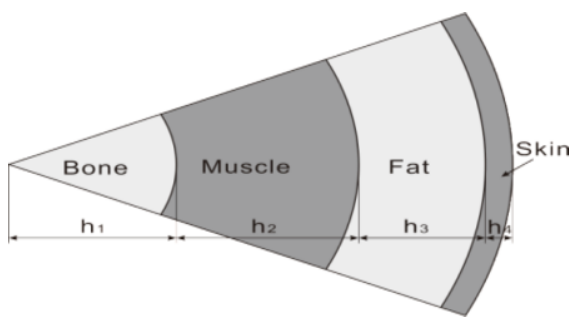

Fig. 3. Structure and size of the human limbs in the simulation.

Table 2

Current density in specific layers of upper limb (all units in $\mathrm{A} / \mathrm{m}^{2}$ )

\begin{tabular}{lllll}
\hline Frequency & $300^{\text {th }}$ & $900^{\text {th }}$ & $1280^{\text {th }}$ & $1368^{\text {th }}$ \\
\hline $0 \mathrm{~Hz}$ & 0.0509 & 0.2546 & 0.0187 & 0.0006 \\
$10 \mathrm{kHz}$ & 0.0509 & 0.2546 & 0.0167 & 0.0029 \\
$100 \mathrm{kHz}$ & 0.0509 & 0.2546 & 0.0146 & 0.0038 \\
$1 \mathrm{MHz}$ & 0.0509 & 0.2546 & 0.0122 & 0.1693 \\
$10 \mathrm{MHz}$ & 0.0509 & 0.2546 & 0.0106 & 0.1698 \\
$1 \mathrm{GHz}$ & 0.0509 & 0.1643 & 0.0075 & 0.2651 \\
\hline
\end{tabular}

The assumed values are as follows: $h_{1}=15 \mathrm{~mm}, h_{2}=15 \mathrm{~mm}, h_{3}=4 \mathrm{~mm}$, and $h_{4}=0.4 \mathrm{~mm}$, and the length of $\operatorname{limb} l=400 \mathrm{~mm}$. The human body, given its relative magnetic permeability of $\mu_{r}=1$, is nonmagnetic and its magnetic permeability $\mu=\mu_{r} \times \mu_{0}=4 \pi \times 10^{-7} \mathrm{H} / \mathrm{m}\left(\mu_{0}\right.$ is the magnetic permeability of vacuum). The conductivity values of different tissues at different frequencies were based on the data presented in Table 1. In the simulation, the cylindrical limb conductor was divided into $N=1376$ concentric rings with same thickness. The $1^{\text {st }}$ to $600^{\text {th }}$ layers represent bone tissues, the $601^{\text {st }}$ to $1200^{\text {th }}$ layers represent muscular tissues, the $1201^{\text {st }}$ to $1360^{\text {th }}$ layers represent fat, and the $1361^{\text {st }}$ to $1376^{\text {th }}$ layers represent skin.

Assuming that the valid value of current density of the first layer $\left(J_{1}\right)$ is $10^{-10} \mathrm{~A} / \mathrm{m}^{2}$, the valid value of current density of the second layer $J_{2}$ was calculated using Eq. (12) and density equation. In addition, the valid values of current density of the other layers were obtained using recurrence Eq. (11) anddensity equation. Simulation results at different frequencies are shown in Figure 4, in which the horizontal axis represents the divided layer quantities from inside to outside, whereas the vertical axis represents the corresponding valid current density values. The valid current density values of the $300^{\text {th }}$, $900^{\text {th }}$, and $1368^{\text {th }}$ layer are shown in Table 2.

The frequencies of $0 \mathrm{kHz}, 10 \mathrm{kHz}, 100 \mathrm{kHz}, 1 \mathrm{MHz}, 10 \mathrm{MHz}$, and $1 \mathrm{GHz}$ were employed in this study, as shown in Figure 4. Under the action of AC with frequency ranging between 0 and $10 \mathrm{MHz}$, the current of each tissue is uniformly distributed. Under the action of DC, the distribution of current density is only related to the conductivity of the tissue. With the increase in frequency, the current density of skin increases, whereas the current density of bone, muscle, and fat are relatively stable, indicating that the skin have exhibited the skin effect, which becomes evident with the increase in frequency. When the frequency of $\mathrm{AC}$ reaches $1 \mathrm{GHz}$, the tissues that are distant from the axis present obvious skin effect, where the current density is unevenly distributed inner portions of the same tissue and density is higher near the tissue surface. 


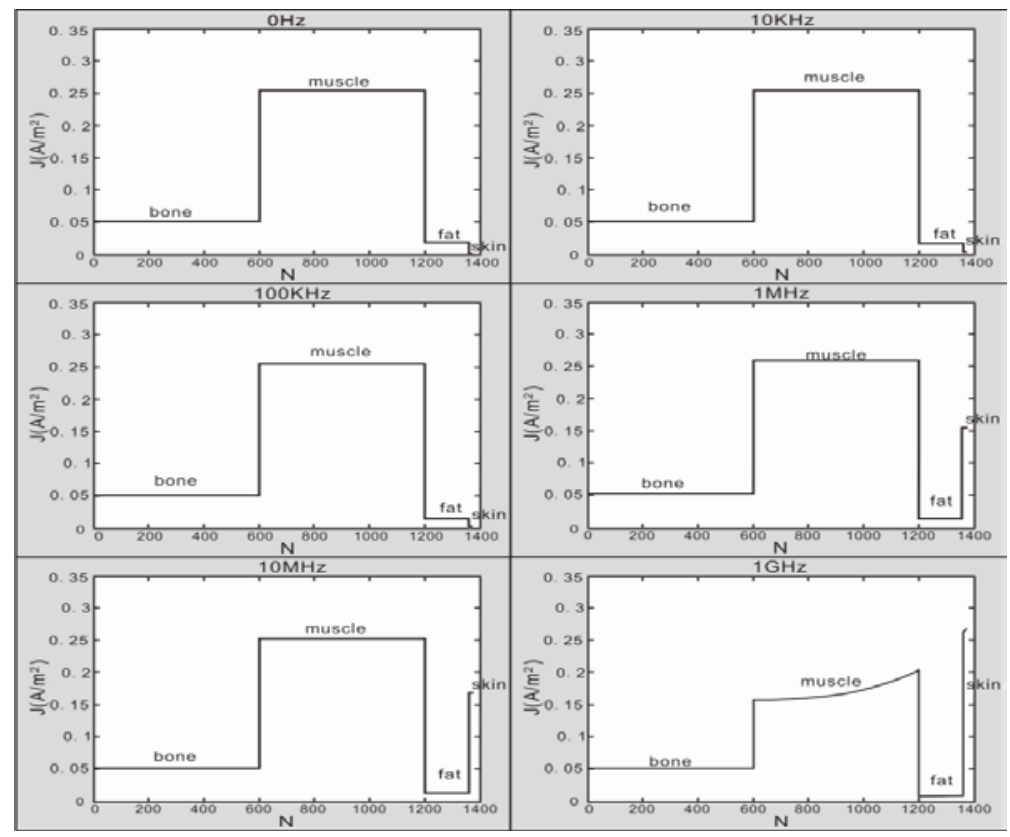

Fig. 4. Simulation results of the current density distribution of human limb at different frequencies.

The current densities of the limb model in different depths are shown in Table 2 . The $300^{\text {th }}, 900^{\text {th }}$, $1280^{\text {th }}$, and $1368^{\text {th }}$ layers are the midpoint of bone, muscle, fat, and skin, respectively. When the frequency of the applied $\mathrm{AC}$ ranged between $0 \mathrm{MHz}$ and $10 \mathrm{MHz}$, the current density of bone is constant at $0.0509 \mathrm{~A} / \mathrm{m}^{2}$ and that of muscle is at $0.2546 \mathrm{~A} / \mathrm{m}^{2}$. By comparison, the current density of fat decreases and that of the skin increases from $0.0006 \mathrm{~A} / \mathrm{m}^{2}$ to $1.4885 \mathrm{~A} / \mathrm{m}^{2}$. These findings indicate that the current transfers from fat into skin because of skin effect. When the frequency reaches $1 \mathrm{GHz}$ (i.e., the threshold value), current distribution is dominated by skin effect, thereby resulting in the maximum outer current density.

For a current flowing through the complex human structure, the current density of DC is only related to conductivity, whereas that of $\mathrm{AC}$ is influenced by skin effect to a certain extent. When the frequency of $\mathrm{AC}$ reaches the threshold value, skin effect exerts considerable influence on density distribution. In bioimpedance measurement, the change in bioimpedance is the basic foundation for pathological diagnosis. In traditional measuring methods, such as two-electrode and four-electrode systems, AC is applied into the target by using an electrode in the skin, and then voltage is received by the detection electrode. Skin effect must be considered in selecting a proper frequency to reduce this influence. These methods are unsuitable for use at excessively high frequencies.

\section{Conclusion}

Bioimpedance measurement technology is widely used in clinical medicine, in which high accuracy of measurement is necessary. The layer-dividing algorithm proposed in this paper has solved the difficulties encountered in studies of the current distribution of complex structures. The layer-dividing algorithm produces accurate results, by which the existence of skin effect in complex human structures under high AC frequency is verified. The algorithm is substantially simpler than finite element method 
with regard to programming and calculation. However, there are still some potential limitations for this algorithm. Firstly, it is only applied in the constructions with regular shapes, but most of human structures are irregular and their compositions are not homogeneous and continuous. Secondly, the self-skin-effect of each layer was not considered in this study. Further studies should consider all these factors and combine multiple methods to develop a novel model for the simulation of skin effect in biological tissue.

\section{Acknowledgments}

This research was funded by National Natural Science Fund (31170895), National Defence preresearch Fund (A0920132003), and Human Factors Engineering Key Laboratory Fund Project (HF2013-K-06).

\section{References}

[1] C.S. Ren, Measurement Technology of Bioelectrical Impedance, Breast Disease 10 (2004), 21-25.

[2] W.R. Ze, X.R. Gao and Q. Ouyang, Multi-channel independent source bio-impedance measurement and signal analysis, Space Medicine \& Medical Engineering 14 (2001), 34-36.

[3] C.G. Yuan, Mixing frequency bioelectrical impedance measuring system, Master Dissertation, Tianjin University, 2005.

[4] S. Grimmes and O.G. Martinsen, Bio-Impedance and Bio-Electricity Basis, Elsevier Academic Press, Boston, 2008 , pp. 123-135

[5] J.D. Jackson, Classical Electrodynamimcs, 3rd ed., John Wiley \& Sons Inc., New York, 1999.

[6] P. Molfino, M. Repetto and R. Sestero, Skin effect analysis in axisymmetric stratified conductors, IEEE Transactions on Magnetics 26 (2009), 721-730.

[7] Z. Liu. Skin effect in proton exchange membrane fuel cell, Master Dissertation, Wuhan University of Technology, 2010.

[8] Q. Wang and H. Xu, Research Progress on measurement technology and clinical application of bioimpedance, Beijing Biomedical Engineering 33 (2014), 185-189.

[9] H. Keren and D. Burkhoff, Evaluation of a noninvasive continuous cardiac output monitoring system based on thoracic bioreactance, American Journal of Physiology. Heart and Circulatory Physiology 293 (2007), 583-589.

[10] G.J. Saulnier, R.S. Blue, J.C. Newell, et al., Electrical impedance tomography, IEEE Signal Processing Magazine 18 (2001), 31-43

[11] H. Zhao, Modeling of a motor in high frequency in the case of data transmission by power line, Master Dissertation, Ocean University of China, 2008.

[12] C. Gabriel, S. Gabriel and E. Corthout, The dielectric properties of biological tissues: Literature survey, A Physics in Medicine \& Biology 41 (1996), 2231-2249.

[13] C.S. Ren, Medical electrical impedance technology and breast tumor measurement, China Medical Device Information 8 (2002), 24-27.

[14] P. Metherall, Three dimensional EIT of the human thorax, Ph.D. Dissertation, University of Sheffield, 1998.

[15] D.C. Wang, Electromagnetic Field Theory and Microwave Technology, Science Press, 1986.

[16] G. Coatache, Finite element method applied to skin effect problems in strip transmission line, IEEE Transactions on Microwave Theory and Techniques 35 (1987), 1009-1013.

[17] W. He, Z. Xu and C.H. He, Parsing algorithm for the potential distribution of head sphere model inspired by point current source, Eleventh China Electrotechnical Mathematics Annual Conference Proceedings, Fuzhou, China, 2007, 19-27.

[18] S. Rush, D.A. Driscoll, Current distribution in the brain from surface electrodes, Anesthesia Analgesia 47 (1968), 717.

[19] A.J. IIott and S. Chandrashekar, Visualizing skin effects in conductors with MRI: Li MRI experiments and calculations, Journal of Magnetic Resonance 245 (2014), 143-149.

[20] T.F. Oostendorp, J. Delbeke and D.F. Stegeman, The conductivity of the human skull: Results of in vivo and in vitro measurements, IEEE Transactions on Biomedical Engineering 42 (2000), 1487. 